\title{
SURGICAL OPTIONS IN PERIPROSTHETIC FRACTURES
}

\author{
doi: 10.2478/rojost-2018-0032 \\ D. Popescu ${ }^{1}$, R. Nedelcu' ${ }^{1}$, St. Trifu ${ }^{1}$, C. Cîrstoivi, ${ }^{12}$ \\ ${ }^{1}$ Orthopedics and Traumatology Department, University Emergency Hospital, Bucharest, Romania \\ "Carol Davila" University of Medicine and Pharmacy, Bucharest, Romania
}

Purpose. The periprosthetic fractures are a more and more often encountered type of pathology, in which the main problem is the indication for surgery. The most important thing is the choice of the best therapeutic option in order to get a solid fixation of the fracture and, in the end, to allow an early patient's mobilization.

Materials and method. 38 cases of periprosthetic fractures have been treated in the Orthopedics and Traumatology Department of University Emergency Hospital in Bucharest, between 2010 and 2016. International Vancouver classification was used for all cases. The osteosynthesis saving the femoral stem was preferred in 22 cases, as its stability was not affected. Stem revision was performed in 16 cases, as this was unstable due to the fracture. Acetabular component was also revised in 4 cases, as the PE insert presented severe wear. The patients were aged 52 to 84 years old and sex ratio M/ F = 13/25. Osteosynthesis was performed using Dall-Miles plates and molded plates, with braided cables or wire cerclage. Long stems, uncemented with or without distal locking, were used in 16 cases.

Results. Postoperatively, the bone repair was efficient regarding the stability in most of the cases. The patients' mobilization was early in most of the cases, except for the very old patients with associated comorbidities and limited biological resources.

Conclusions. An appropriate surgical indication, adapted on each type of peri-implant fracture, leads to a good result, with early mobilization and the best consolidation of the fracture.

Keywords: periprosthetic, osteosynthesis, revision, consolidation, mobilization 